\title{
The usefulness of CEA and/or CA19-9 in monitoring for recurrence in gastric cancer patients: a prospective clinical study
}

\author{
Yutaka Takahashi, Tetsuo Takeuchi, Junichi Sakamoto, Tetsuya Touge, Masayoshi Mai, Hisanao Ohkura, \\ Susumu Kodaira, Kunio Okajima, and Hiroaki Nakazato; Tumor Marker Committee \\ Japanese Foundation for Multidisciplinary Treatment of Cancer, Ichigaya MS Bldg., 1-9, 4-Chome, Kudankita, Chiyoda-ku, Tokyo 102-0073, \\ Japan
}

\begin{abstract}
Background. Many studies on postoperative carcinoembryonic antigen (CEA) and/or carbohydrate antigen (CA)19-9 monitoring after operation for gastric cancer have been reported, but most have been retrospective.

Methods. A nationwide observational study was implemented in 135 leading institutions in Japan to evaluate the significance of CEA and/or CA19-9 in postoperative monitoring for recurrence in patients with advanced gastric cancer. Three hundred and twenty-one patients examined in this analysis underwent radical gastrectomy at one of Japan's leading institutions between November 1993 and March 1996 and had been followed up for at least 5 years. Serum levels of CEA and CA19-9 were examined preoperatively and every 3 months postoperatively, with diagnostic imagings, such as chest X-ray, computed tomography (CT), and ultrasonography also being performed every 3 months.

Results. Recurrence was observed in 120 patients (peritoneum, 48; liver 16; lymph node, 16; multiple sites, 25; and others, 12). Sensitivities of CEA and either CEA or CA19-9, or both, for recurrence were $65.8 \%$ and $85.0 \%$, respectively, both of which values were significantly higher than the preoperative positivities ( $28.3 \%$ and $45.0 \%$, respectively). In most patients with high preoperative levels CEA and/or CA19-9, these tumor markers increased again at recurrence. Recurrent diseases were detected between 5 months after detection by diagnostic imagings and 12 months before detection by diagnostic imagings (mean of 3.1 \pm 3.6 months before detection by diagnostic imagings) and between 10 months after detection by diagnostic imagings and 13 months before detection by diagnostic imagings (mean of $2.2 \pm 3.9$ months before detection by diagnostic imagings) by CEA and CA19-9 monitorings, respectively.

Conclusion. These results suggest that CEA and/or CA19-9 monitoring after operation was useful to predict the recurrence of gastric cancer, especially in almost all the patients with high preoperative levels of these markers.
\end{abstract}

Offprint requests to: Y. Takahashi Received: January 23, 2003 / Accepted: April 21, 2003 We reported this study at the poster session of the eighteenth meeting of the American Society of Clinical Oncology.
Key words Gastric cancer · Recurrence $\cdot$ CEA · CA19-9

\section{Introduction}

Although there are no specific tumor-associated antigens in gastric cancers, carcinoembryonic antigen (CEA) and carbohydrate antigens of the sialyl-Lewis A group, such as CA19-9, are known to be elevated in the serum of patients with advanced gastric cancer. A combination of CEA and CA19-9 monitoring can extend the possibilities of their use for the early detection for recurrence after operation for advanced gastric cancer. Although many studies of postoperative CEA and/or CA19-9 monitoring for gastric cancer have been reported [1-8], the usefulness of this monitoring is still controversial. Because most of these studies have been retrospective and small-scale and because there were different monitoring periods in the patients, we implemented a nationwide observational study to evaluate the usefulness of CEA and/or CA19-9 in postoperative monitoring for recurrence in patients with advanced gastric cancer. This was a prospective study carried out as an additional study of adjuvant chemotherapy for far-advanced gastric cancer.

\section{Patients and methods}

Three hundred and twenty-one patients with advanced gastric cancer of more than stage II, according to the Japanese Research Society for Gastric Cancer [9], examined in this analysis underwent radical gastrectomy at one of 135 leading Japanese institutions between November 1993 and March 1996: they were followed for at least 5 years in the JFMTC (Japanese Foundation for Multidisciplinary Treatment of Cancer) study no. 20 [10], a randomized control study comparing intensive 
Table 1. Characteristics of 120 patients with recurrence

\begin{tabular}{llr} 
Male/Female & $77 / 43$ & \\
Age in years (median) & $43-75(59)$ & \\
Stage of disease & a & $16(13.3 \%)$ \\
& b & $87(72.5 \%)$ \\
Gross type & a & $17(14.2 \%)$ \\
& 0 & $3(2.5 \%)$ \\
& 1 & $0(0.0 \%)$ \\
Histological type & 2 & $16(13.3 \%)$ \\
& 3 & $67(55.8 \%)$ \\
& 4 & $34(28.3 \%)$ \\
& Pap & $2(1.7 \%)$ \\
& Tub1 & $6(5.0 \%)$ \\
Tub2 & $22(18.3 \%)$ \\
& Por1 & $22(18.3 \%)$ \\
& Por2 & $47(39.2 \%)$ \\
& Sig & $16(13.3 \%)$ \\
& Muc & $5(4.2 \%)$ \\
& E & $1(0.8 \%)$ \\
& C & $20(16.7 \%)$ \\
& M & $51(42.5 \%)$ \\
& A & $48(40.0 \%)$ \\
\hline
\end{tabular}

$\overline{\text { E, esophagus; C, cardia; M, middle; A, antrum; Pap, papillary adeno- }}$ carcinoma; Tub, tubular adenocarcinoma; Por, poorly differentiated adenomcarcinoma; Sig, signet-ring cell adenocarcinoma; Muc, mucinous adenocarcinoma

and standard adjuvant chemotherapy. We obtained informed consent from all patients for this protocol. Serum levels of CEA and CA19-9 were examined preoperatively and every 3 months postoperatively. Diagnostic imagings such as chest X-ray, computed tomography $(\mathrm{CT})$, and ultrasonography were also performed every 3 months, to check for recurrence, for at least 3 years. Recurrence was observed in 120 patients during the 3 years after operation (peritoneum, 48; liver, 16; lymph node, 16; multiple sites, 25; and others, 12). Their whose characteristics are shown in Table 1.

First we studied the preoperative positivities and sensitivities of CEA and CA19-9 for the patients with recurrence. Next, we examined the longitudinal changes in CEA and CA19-9 positivities at the preoperative and recurrent stages in individual patients, and we also studied the specificity (true-negatives/true-negatives + false-positives) for recurrent stages in 95 patients who had no recurrence at 5 years after surgery. Lastly, we studied the lead time of CEA or CA19-9 compared with the follow-up findings obtained by using imagings. The lead time was calculated as the time of first detection of elevated markers postoperatively subtracted from the time of first recurrence indicated by chest X-ray, CT, or Echo imagings. A positive lead time indicated that markers were elevated earlier than the recurrence noted by imagings. We also compared the lead time in patients with higher and lower preoperative marker levels (higher CEA group, greater than $10 \mathrm{ng} / \mathrm{ml}$; higher CA19-9 group, greater than $70 \mathrm{IU} / \mathrm{ml}$ ).
Table 2. Comparative preoperative positivities for CEA and CA19-9 values and their sensitivities for indicating recurrence

\begin{tabular}{lcr}
\hline & $\begin{array}{c}\text { Preoperative } \\
\text { positivities }\end{array}$ & $\begin{array}{c}\text { Sensitivities } \\
\text { for recurrence }\end{array}$ \\
\hline CEA & $34(28.3 \%)$ & $79(65.8 \%)$ \\
CA19-9 & $35(29.2 \%)$ & $66(55.0 \%)$ \\
Either/both & $54(45.0 \%)$ & $102(85.0 \%)$ \\
\hline
\end{tabular}

CEA, carcinoembryonic antigen; $\mathrm{CA}$, carbohydrate antigen

Student's $t$-test and the $\chi^{2}$ test were used to study the differences between positivities for preoperative CEA and/or CA19-9 and their sensitivities for indicating recurrence, differences in the lead time between the higher and lower CEA and CA19-9 groups, and differences between liver and other recurrence sites.

\section{Results}

Preoperative positivities for CEA and CA19-9

and their sensitivities and specificities for indicating recurrence

Sensitivities for CEA and CA19-9, and combinations of the two markers, for indicating recurrence were $65.8 \%$, $55.0 \%$, and $85.0 \%$, all of which values were significantly higher than the preoperative positivities $(28.3 \%, 29.2 \%$, and $45.0 \%$, respectively; $P<0.001$ for all; Table 2). These results suggest that the usefulness of serum CEA and CA19-9 values is much greater for indicating recurrence than for indicating metastases preoperatively. Moreover, the specificities of serum CEA and CA19-9 for recurrence were $81.1 \%$ and $93.7 \%$, respectively.

\section{Longitudinal changes in CEA and CA19-9 positivities at the preoperative and recurrent stages in individual patients}

Of the 34 patients with elevated preoperative levels of CEA, this marker increased again at recurrence in $32(94.1 \%)$ patients. Similarly, CA19-9 was increased again at recurrence in $32(91.4 \%)$ of the 35 patients with high preoperative levels of this marker. There were also many patients whose CEA and CA19-9 levels increased for the first time at recurrence $(54.7 \%$ and $40.0 \%$, respectively; Table 3). These data indicate that we can detect elevated levels of serum CEA or CA19-9 at recurrence in more than $90 \%$ of patients with high preoperative levels of these markers.

\section{Lead-time determinations of CEA and CA19-9 levels based on imaging results}

The lead times were -5 to 12 months, with a mean of $3.1 \pm 3.6(\mathrm{SD})$ months, and -10 to 13 months, with 
a mean of $2.2 \pm 3.9$ months for CEA and CA19-9 monitorings, respectively (Table 4). In the higher CEA group, the lead times were 1 to 12 months, with a mean of $5.2 \pm 2.2$ months, and the values were -5 to 4 months, with a mean of $-1.3 \pm 2.8$ months, in the lower CEA group. In the higher CA19-9 group, these values were -2 to 13 months, with a mean of $4.1 \pm 2.7$ months, and in the lower CA19-9 group, and the values were -10 to 7 months, with a mean of $0.5 \pm 3.1$ months. Significant differences in the lead times were observed between the higher groups and lower groups for both markers $(P<0.001$ for both) (Table 5$)$.

Table 3. Longitudinal changes in CEA and CA19-9 positivities at the preoperative and recurrent stages in individual patients

\begin{tabular}{lcccc}
\hline $\begin{array}{l}\text { Preoperative } \\
\text { positivities }\end{array}$ & $\begin{array}{c}\text { Sensitivities } \\
\text { for recurrence }\end{array}$ & $\begin{array}{c}\text { CEA } \\
n\end{array}$ & $\begin{array}{c}\text { CA19-9 } \\
n\end{array}$ \\
\hline- & $\longrightarrow$ & + & 47 & 34 \\
+ & $\longrightarrow$ & + & 32 & 32 \\
- & $\longrightarrow$ & - & 39 & 51 \\
+ & $\longrightarrow$ & - & 2 & 3 \\
\hline
\end{tabular}

Table 4. Comparison of the lead times for CEA and CA19-9 levels and the imaging results

\begin{tabular}{lcc}
\hline & $\begin{array}{c}\text { Lead time } \\
\text { compared with imagings }\end{array}$ & $\begin{array}{c}\text { Mean } \pm \text { SD } \\
\text { of the lead time }\end{array}$ \\
\hline CEA & -5 to 12 Months & $3.1 \pm 3.6$ Months \\
CA19-9 & -10 to 13 Months & $2.2 \pm 3.9$ Months \\
\hline
\end{tabular}

We next studied the differences in lead times among recurrent sites. The lead times for recurrences in the liver, peritoneum, and lymph nodes were $1.2 \pm 4.0,3.4$ \pm 3.8 , and $3.7 \pm 3.5$ months, respectively, for CEA; and $2.1 \pm 2.6,1.0 \pm 4.3$, and $3.6 \pm 4.0$ months, respectively, for CA19-9. The lead time for CEA for liver recurrence was significantly shorter than those for the peritoneum and lymph node metastases $(P<0.05$; Table 6$)$.

\section{Discussion}

The usefulness of serum tumor markers is still controversial. According to the guidelines of the American Society of Clinical Oncology [11], the serum CEA level is not recommended for screening or for use as a surrogate marker of chemotherapy, but it is recommended for the preoperative prediction of disease staging and for monitoring recurrence after surgery in colon cancer. However, it is not recommended for the screening, diagnosis, staging, or routine surveillance of breast cancer patients after operation. In the present prospective study, we examined whether the serum CEA level could be recommended for monitoring the recurrence of gastric cancer after surgery.

Many tumor markers, such as CEA, CA19-9, CA724, CA12-5, and CA-50, have been studied in the monitoring of gastric cancer [1-8], but the sensitivity of any one of them is not sufficient. Our study showed that preoperative positivities for CEA, and CA19-9, and their combination were $28.3 \%, 29.2 \%$, and $45.0 \%$, respectively, in 321 patients with moderately advanced gastric cancers. However, the sensitivities for recur-

Table 5. Lead times for higher and lower CEA and CA19-9 groups

\begin{tabular}{|c|c|c|c|}
\hline & $\begin{array}{l}\text { Lead time compared } \\
\text { with imagings }\end{array}$ & \multicolumn{2}{|c|}{$\begin{array}{c}\text { Mean } \pm \mathrm{SD} \\
\text { of the lead time }\end{array}$} \\
\hline \multicolumn{4}{|l|}{ CEA } \\
\hline higher group $(n=43)$ & 1 to 12 Months & $5.2 \pm 2.2$ Months & \multirow{2}{*}{$P<0.001$} \\
\hline Lower group $(n=36)$ & -5 to 4 Months & $-1.3 \pm 2.8$ Months & \\
\hline \multicolumn{4}{|l|}{ CA19-9 } \\
\hline Higher group $(n=38)$ & -2 to 13 Months & $4.1 \pm 2.7$ Months & \multirow{2}{*}{$P<0.001$} \\
\hline Lower group $(n=28)$ & -10 to 7 Months & $0.5 \pm 3.1$ Months & \\
\hline
\end{tabular}

Table 6. Differences in lead times among recurrent sites

\begin{tabular}{lcc}
\hline & CEA & CA19-9 \\
\hline Liver $(n=16)$ & $1.2 \pm 4.0$ Months \\
Peritoneum $(n=48)$ & $3.4 \pm 3.8$ Months $]_{*}$ & $2.1 \pm 2.6$ Months \\
Lymph node $(n=16)$ & $3.7 \pm 3.5$ Months $]_{*}$ & $1.0 \pm 4.3$ Months \\
\hline
\end{tabular}

$* P<0.05$ 
rence were $65.8 \%, 55.0 \%$, and $85.0 \%$, respectively, in 120 patients with recurrence. These significant increases in sensitivity suggest that the serum levels of these markers would be highly suitable for monitoring for recurrence after operation. Moreover, in more than $90 \%$ of patients with high preoperative levels of CEA or CA19-9, these markers increased again at recurrence. In other words, we can follow up on recurrence by monitoring these tumor markers in patients with high preoperative levels of these markers.

We also examined whether or not we could detect recurrence earlier by the monitoring of the tumor markers than by imagings. If we cannot detect recurrences when they are of a smaller size than recurrences detected by imagings, monitoring by tumor markers is not very useful. When we perform imagings after the elevation of tumor marker levels, we may detect large-size recurrences. Therefore, we determined which was the earlier event, detection of recurrence by imaging, or the elevation of tumor markers, by studying the lead time of tumor markers based on imaging. Recurrent diseases were detected at -5 to 12 months (mean, $3.1 \pm 3.6$ (SD) months) for CEA monitoring and at -10 to 13 months (mean, $2.2 \pm 3.9$ months) for CA19-9 monitoring before detection by imaging. These results indicate that monitoring for recurrence by tumor markers is useful but not sufficient. We need to recognize that elevation of tumor marker levels is seen much later than detection of recurrence by imaging in some cases.

We finally analyzed in which patients the tumor markers were elevated earlier. In the patients with higher preoperative levels of tumor markers, these markers were elevated earlier. We studied the lead times in relation to other factors, such as recurrent sites and pathological classification; the lead time for CEA in liver recurrence was significantly shorter than those for peritoneum and lymph node metastasis $(P<0.05)$, and this was the only significant difference in lead time in relation to other factors.

In summary, monitoring for recurrence by serum CEA and/or CA19-9 levels is useful in most patients with gastric cancers. In particular, in almost all of the patients with high preoperative levels of of the tumor markers, levels of these markers were elevated earlier than the detection of recurrence by some imagings. However, suggest that we should monitor imagings mainly, rather than monitoring these markers, in the remaining patients with low preoperative levels of these markers.

\section{References}

1. Kodama I, Koufuji K, Kawabata S, Tetsu S, Tsuji Y, Takeda J, et al. The clinical efficacy of CA 72-4 as serum marker for gastric cancer in comparison with CA19-9 and CEA. Int Surg 1995;80: $45-8$.

2. Safi F, Kuhns V, Beger HG. Comparison of CA 72-4, CA 19-9 and CEA in the diagnosis and monitoring of gastric cancer. Int J Biol Markers 1995;10:100-6.

3. Guadagni F, Roselli M, Cosimelli M, Ferroni P, Spila A, Casaldi $\mathrm{V}$, et al. Correlation between positive CA 72-4 serum levels and lymph node involvement in patients with gastric carcinoma. Anticancer Res 1993;13:2409-13.

4. Kim YH, Ajani JA, Ota DM, Lynch P, Roth JA. Value of serial carcinoembryonic antigen levels in patients with resectable adenocarcinoma of the esophagus and stomach. Cancer 1995;75: 451-6.

5. Pectasides D, Mylonakis A, Kostopoulou M, Papadopoulou M, Triantafillis D, Varthalitis J, et al. CEA, CA 19-9, and CA-50 in monitoring gastric carcinoma. Am J Clin Oncol 1997;20:348-53.

6. Ohkura H. Tumor markers in monitoring response to chemotherapy for patients with gastric cancer. Jpn J Clin Oncol 1999; 29:525-6.

7. Yamao T, Kai S, Kazami A, Koizumi K, Handa T, Takemoto N, et al. Tumor markers CEA, CA19-9 and CA125 in monitoring of response to systemic chemotherapy in patients with advanced gastric cancer. Jpn J Clin Oncol 1999;29:550-5.

8. Ychou M, Duffour J, Kramar A, Gourgou S, Grenier J. Clinical significance and prognostic value of CA72-4 compared with CEA and CA19-9 in patients with gastric cancer. Dis Markers 2000;16: $105-10$.

9. Japanese Gastric Cancer Association. Japanese classification of gastric carcinoma. 2nd English Ed. Gastric Cancer 1998;1:10-24.

10. Touge T, Fujita M, Hirata K, Kunii Y, Kitamura M, Nagawa K, et al. Interim report of JFMTC study no. 20 on the effectiveness of high dose CDDP plus 5-FU regimen as an adjuvant therapy for far-advanced cancer of the stomach. Gan To Kagaku Ryoho (Jpn J Cancer Chemother) 2000;27:395-403.

11. Bast RC, Ravdin P, Hayes DF, Bates S, Fritsche H, Jesup JM, et al. 2000 Update of recommendations for the use of tumor markers in breast and colorectal cancer: clinical practice guidelines of the American Society of Clinical Oncology. J Clin Oncol 2001;19: 1865-78. 